\title{
A multi-wavelength search for bulge millisecond pulsars
}

\author{
Joanna Berteaud, ${ }^{a, b, *}$ Francesca Calore $^{a}$ and Maïca Clavel ${ }^{b}$ \\ ${ }^{a}$ Univ. Grenoble Alpes, USMB, CNRS, LAPTh, F-74940 Annecy, France \\ ${ }^{b}$ Univ. Grenoble Alpes, CNRS, IPAG, F-38000 Grenoble, France \\ E-mail: berteaud@lapth.cnrs.fr, calore@lapth.cnrs.fr, \\ maica.clavel@univ-grenoble-alpes.fr
}

More than a decade after its discovery, the Fermi $\mathrm{GeV}$ excess is still an exciting subject of research. Thus far, an unresolved population of millisecond pulsars (MSPs) in the Galactic bulge shining in gamma rays is the favorite explanation to the excess, but other explanations exist. Data from the Fermi-LAT have been thoroughly studied and, in order to discriminate between the different hypotheses, a multi-wavelength approach is now needed. In a recent study [1], we demonstrated that if the $\mathrm{GeV}$ excess is caused by an MSP population, about a hundred of them could be detectable in X-rays in a region of $6^{\circ} \times 6^{\circ}$ about the Galactic Center. The comparison with $\mathrm{X}$-ray data allowed us to conclude that the MSP hypothesis was not excluded, as we found more than 3000 MSP candidates in a conservative approach. Besides, we selected few hundreds of promising candidates, with good X-ray spectral knowledge and no optical counterpart. In our new study, we additionally exploit ultraviolet and infrared data to exclude candidates. Finally, we compute a relation between the X-ray and radio luminosity of MSPs, aiming at predicting the radio luminosity of our candidates, with the ultimate goal of motivating radio observations needed to detect a pulsation and confirm a pulsar detection.

$37^{\text {th }}$ International Cosmic Ray Conference (ICRC 2021)

July 12 th $-23 r d, 2021$

Online - Berlin, Germany

\footnotetext{
${ }^{*}$ Presenter
} 


\section{Introduction}

The Fermi Large Area Telescope (Fermi-LAT), launched more than a decade ago, has produced the most detailed $\gamma$-ray data to date. Its energy range and spatial resolution showed undeniable progress compared to its predecessor, EGRET. One objective of the Fermi-LAT was to investigate the composition of the dark matter (DM), and when an excess of $\gamma$ rays around $2 \mathrm{GeV}$ was detected in the direction of the Galactic Center, the scientific community naturally got really excited. Straight away, this signal was interpreted as a possible sign of DM annihilation. However, after more than ten years of research, scientists now favor a more astrophysical explanation: an unresolved population of millisecond pulsars (MSPs) hiding in the Galactic bulge. These sources of $\gamma$-rays, because too faint, would not be resolved as point sources by the Fermi-LAT, but would contribute to the diffuse emission. The spectral shape of the excess, renamed Fermi GeV excess or Galactic Center excess, resembles the one of some globular clusters expected to host MSPs and its spatial morphology follows the stellar over-density of the Galactic bulge. The Fermi-LAT $\gamma$-ray data have been thoroughly studied in order to understand the Fermi GeV excess, and a multi-wavelength approach is now needed in order to discriminate between the different hypotheses.

In a recent study [1], we analysed the sensitivity of the Chandra X-ray observatory to an MSP bulge population. We created a synthetic population of MSPs whose properties, spectral shape and spatial morphology, match the ones of the Fermi GeV excess. We found that $95 \pm 9$ simulated MSPs in a region of interest of $6^{\circ} \times 6^{\circ}$ about the Galactic Center would be detectable by Chandra. Although completely unresolved in $\gamma$ rays, the MSP population could partly be resolved in X rays. Motivated by this unforeseeable discovery, we looked for MSP candidates among the unidentified Chandra sources. We selected candidates based on their distance via their possible Gaia counterparts [2] and $\mathrm{X}$-ray spectral properties so that these characteristics are consistent with the simulated detectable MSP population. In a strictly conservative approach, we found more than 3000 candidates in the same region of interest. Finding less candidates than the number of detectable MSPs in our simulation would have excluded our model, and finding much more is not surprising, knowing the possible contamination of our selection by active galactic nuclei (AGN), chromospherically active stars, quiescent low-mass X-ray binaries and cataclysmic variables (CVs). Among this selection, we then chose the most promising MSP candidates as the ones with a good spectral knowledge and no possible Gaia counterpart at all in [16], regardless of the distance. That way, we retained 203 MSP candidates ${ }^{1}$ interesting for follow-up studies.

In this work, we aimed at further reducing our selection of promising candidates and motivating radio observations of part of the remaining sources. In section 2, we describe the cross-matches we performed between our 203 Chandra sources and data collected by different instruments at various wavelengths. In section 3, we present how we computed a relation between the X-ray and the radio luminosity of MSPs from observational data. Finally in section 4, we present future prospects and conclude.

\footnotetext{
${ }^{1}$ Using the Gaia distance catalog [2], we retained 285 candidates as main result in our previous study [1].
} 


\section{Multi-wavelength cross-matches}

If the Fermi GeV excess is caused by a population of MSPs, these sources must be located in the Galactic bulge, between about 5 and $12 \mathrm{kpc}$ [1]. At these distances, it is very unlikely that an optical counterpart can be detected, as suggested by the low number of MSPs (in binary systems) seen in the Gaia data. Moreover, even though these MSPs are less than $3 \mathrm{kpc}$ away from us, they are already very $\mathrm{dim}$. Selecting as promising MSP candidates only Chandra sources with no optical counterpart was therefore judicious. Moreover, most objects emitting in $\mathrm{X}$ rays (foreground stars, CVs, AGN) are also bright at other wavelengths, this is why we could also use ultraviolet (UV) and infrared (IR) data for cross-matches. If any counterpart exists, it should be very faint and besides, very absorbed. In this section, we present our cross-match procedure and results between our candidates and UV and IR data and last, we compare these results with the literature.

\subsection{UV cross-matches}

For UV cross-matches, we made use of the latest release of the XMM OM Serendipitous Ultraviolet Source Survey catalogue, XMM-OM-SUSS5.02 [13]. This catalogue contains sources detected by the Optical Monitor (XMM-OM) on board the XMM-Newton observatory. We define a positive cross-match if the angular separation between the Chandra MSP candidate and the XMM-OM source is less than $\sqrt{\text { err_ellipse_r0 } 0^{2}+(2 \times \text { POSERR })^{2}}$ where err_ellipse_r0 is the major radius of the $95 \%$ confidence level position error ellipse of the Chandra MSP candidate ${ }^{3}$ and POSERR is the $68 \%$ uncertainty on the position of XMM-OM source. We assume that POSERR has a Gaussian distribution, so that $2 \times$ POSERR is the $95 \%$ uncertainty on the position. Following these definitions, we find 7 positive cross-matches that reject 7 MSP candidates.

\subsection{IR cross-matches}

Given the increasing sensitivity of IR instruments, the IR counterpart of MSPs, although very faint, could be detectable. It has been shown that compact objects have a high X-ray-to-IR flux ratio, with $\log _{10}\left(F_{X M M} / F_{K}\right)>0.5$ [8] where $F_{X M M}$ is the $0.2-12 \mathrm{keV}$ absorbed X-ray flux seen by XMM and $F_{K}=-k_{s} / 2.5-6.95$ is the infrared flux in the 2MASS $K_{s}$ band in erg $/ \mathrm{cm}^{2} / \mathrm{s}$ and $k_{s}$ is the magnitude in this same band. Using our simulated MSP population, we calculated that the flux seen by XMM $F_{X M M}$ should be at least equal to, and at most 3 times larger than the flux seen by Chandra flux_aper_90_b. If an MSP candidate has no possible IR counterpart or if one is found and the criterion is respected, the candidate is kept. On the contrary, if a counterpart is found and the criterion is not respected, we exclude the candidate from the promising sources.

Knowing this criterion, the first IR data we worked with were naturally 2MASS data ${ }^{4}$ [14]. We neglected the size of 2MASS error ellipses and considered a positive cross-match if the angular distance between a 2MASS source and a Chandra MSP candidate is less than err_ellipse_r0, and found 10 of them. For all ten associations, $\log _{10}\left(3 \times\right.$ flux_aper90_b/ $\left.F_{K}\right) \leq 0$, indicating that none of the sources are compact objects and therefore, that they can all be removed from the candidate selection.

2https://www. cosmos. esa. int/web/xmm-newton/om-catalogue

3https://cxc. harvard. edu/csc2/columns/master_alpha.html

4https: //cdsarc . unistra. fr/viz-bin/cat/II/246 
The second IR data we worked with were VVV data ${ }^{5}$ [12]. We used the same compact object criterion as for 2MASS data, assuming that $K_{s}^{2 M A S S}=K_{S}^{V V V}$. The latter is not exactly true but the difference is negligible here ${ }^{6}$. In order to find all sources with a possible IR counterpart, we define a positive cross-match if the angular separation between a candidate and a VVV source is less than err_ellipse_r0. As for 2MASS, we neglected the size of the error ellipse on VVV data. We found 23 positive cross-matches in total, and for all of them the compact object criterion was not respected.

The last data we used are GLIMPSE data collected with the IRAC aboard the Spitzer Space Telescope ${ }^{7}$ [4]. We found 5 positive cross-matches for which the angular separation between a Chandra source and a GLIMPSE source is less than $\sqrt{\text { err_ellipse_r0 } 0^{2}+(2 \times 0.3)^{2}}$ arcsec. One GLIMPSE source in the positive cross-matches is associated to a 2MASS source and the matching Chandra source was not already excluded as the angular separation between it and the 2MASS source was slightly above err_ellipse_ro. We checked that the compact object criterion was not respected for this source. For the 4 remaining GLIMPSE associations, no 2MASS counterpart was listed, and a detection in mid IR with no near IR counterpart indicates a red-shifted or dusty object, probably outside the Milky Way.

\subsection{Comparison with cross-matches from the literature and summary}

The Chandra Galactic Bulge Survey (GBS) [6] was followed by a number of multi-wavelength studies. A radio counterpart study [9] found a possible pulsar candidate in the GBS, and this Chandra source is also found in our conservative selection of MSP candidates. It was not retained as a promising candidate as it lacks spectral information. Among our VVV-MSP candidate crossmatches, one Chandra source is a GBS source. A near IR counterpart study [5] also found a VVV counterpart for this source.

In summary, we could remove from our promising candidates selection 7 sources with a UV counterpart, 33 with a near IR counterpart (10 with 2MASS and 23 with VVV) and 5 with a mid IR counterpart, leaving us with 158 promising candidates. We looked for radio counterparts to these objects in existing catalogs, but none was found. However, radio emissions would be very helpful to identify MSPs among our candidates.

\section{Radio luminosity predictions}

In order to prove that a source is a pulsar, a pulsation needs to be detected. Given the very faint $\mathrm{X}$-ray flux of our candidates, we have very little hope to detect an X-ray pulsation. However, MSPs also pulse at radio wavelengths. Considering that our candidate MSPs are true MSPs, we would like to predict their radio flux to check that the existing non detection are not ruling out the MSP hypothesis and to motivate deeper radio observations.

5https://cdsarc. unistra.fr/viz-bin/cat/II/348

${ }^{6}$ http://casu.ast.cam.ac.uk/surveys-projects/vista/technical/photometric-properties

7https://cdsarc.unistra.fr/viz-bin/cat/II/293 


\subsection{Data sample}

To make our predictions, we relied on observational MSP data. We used the same list of 47 X-ray detected MSPs [7] as in our previous study [1], and looked for the radio emission of these MSPs in the ATNF pulsar catalog ${ }^{8}$ [11]. All 47 MSPs are found in the ATNF catalog but not all of them have the information that we want provided. In particular, 30 (26) MSPs have $S_{1400}\left(S_{400}\right)$ given, and 18 have both given. $S_{1400}$ and $S_{400}$ are the mean flux densities at 1400 and $400 \mathrm{MHz}$, respectively, in mJy. For MSPs with $S_{1400}$ provided, we define $l_{R}$ as:

$$
l_{R}=\log _{10}\left(S_{1400}\right)+\log _{10}\left(4 \pi d^{2}\right)
$$

where $d$ is the MSP distance (DIST_DM in ATNF). For MSPs with only S400 provided, we define $l_{R}$ as:

$$
l_{R}=\log _{10}\left(S_{400}\right)-\bar{\alpha} \log _{10}(1400 / 400)+\log _{10}\left(4 \pi d^{2}\right)
$$

where the second term on the right hand side corresponds to the conversion from $S_{400}$ to $S_{1400}$. MSPs have a radio power law spectrum, with $S_{v} \propto v^{-\alpha}$. The mean radio index $\bar{\alpha}=1.76$ was averaged over $\alpha$ values of the 18 MSPs having $S_{1400}$ and $S_{400}$ provided:

$$
\alpha=-\frac{\log _{10}\left(S_{1400} / S_{400}\right)}{\log _{10}(1400 / 400)} .
$$

For the 38 MSPs having $S_{1400}$ or $S_{400}$ provided, we define $l_{X}$ as:

$$
l_{X}=l_{\text {Lee }}+\log _{10}\left(\frac{\int_{2-7 \mathrm{keV}} E^{-\Gamma+1} d E}{\int_{2-10 \mathrm{keV}} E^{-\Gamma+1} d E}\right)+\log _{10}\left(\frac{d^{2}}{d_{\text {Lee }}^{2}}\right)
$$

where $l_{\text {Lee }}$ is the $\log _{10}$ of the MSP X-ray unabsorbed flux in the 2-10 keV band and $\Gamma$ the MSP $\mathrm{X}$-ray spectral index from [7], the second term accounts for the conversion from the 2-10 keV band to the 2-7 keV band, and the last term is an adjustment made to use the same MSP distance $d$ in radio as in X-rays, instead of using $d_{\text {Lee }}$ provided in [7] in X-rays.

\subsection{Uncertainties on $\mathrm{X}$-ray and radio luminosities}

The $l_{R}$ and $l_{X}$ computed in section 3.1 are subject to uncertainties. In the ATNF pulsar catalog, the uncertainty on the flux is often provided while the error on the distance is never provided. To compute the latter, we made use of the Python package PSRDist [3] and adapted example2 . py ${ }^{9}$ for our purpose. For each pulsar with a dispersion measurement DM, a latitude and a longitude, the code computes the best-fit distance $d$ using the best-fit model for Galactic electron density [17]. The distance $d$ found equals Dist_DM. The code also computes the distance probability density function (PDF) using Monte Carlo simulations. Parameters of the electron density models are drawn from a Gaussian distribution with uncertainties corresponding to the uncertainty of the best-fit model [17]. That way, the code creates $\mathrm{N}$ electron density models and gets $\mathrm{N}$ distances for each pulsar. Finally, a KDE method is used to obtain the PDF of the distance. We fitted these PDFs with a Gaussian

8http://www.atnf.csiro.au/research/pulsar/psrcat

9https://github.com/tedwards2412/PSRdist/blob/master/examples/example2. py 
distribution, imposing $\mu=d$ and used the best-fit $\sigma$ as error on the pulsar distance, $\Delta d$. Doing so, we find an average value of $7.43 \%$ for $\Delta d / d$. The uncertainty $\Delta l_{R}$ is then:

$$
\Delta l_{R}^{2}=\left(\log _{10}(e) \frac{\Delta S_{1400}}{S_{1400}}\right)^{2}+\left(2 \times \log _{10}(e) \frac{\Delta d}{d}\right)^{2}
$$

when $S_{1400}$ and $\Delta S_{1400}$ are provided. In this case, the average of the term inside the first brackets is 0.109 , and if $S_{1400}$ is provided but $\Delta S_{1400}$ is not, this term is replaced by 0.109 . If only $S_{400}$ is provided, the uncertainty $\Delta l_{R}$ is then:

$$
\Delta l_{R}^{2}=0.061^{2}+\left(\log _{10}\left(\frac{1400}{400}\right) \Delta \alpha\right)^{2}+\left(2 \times \log _{10}(e) \frac{\Delta d}{d}\right)^{2}
$$

where $\Delta \alpha=0.80$ is the standard deviation around $\bar{\alpha}$ and the first term is the mean value of $\log _{10}(e) \frac{\Delta S 400}{S 400}$ for the 18 MSPs with $S_{400}$ and $S_{1400}$ given. We could not compute $\log _{10}(e) \frac{\Delta S_{400}}{S_{400}}$ for MSPs that only have $S_{400}$ provided because for these sources $\Delta S_{400}$ was not provided. The uncertainty $\Delta l_{X}$ associated to the $\mathrm{X}$-ray luminosity is calculated as follows:

$$
\Delta l_{X}^{2}=\max \left(\Delta l_{\text {Lee }}^{+}, \Delta l_{\text {Lee }}^{-}\right)^{2}-\left(2 \times \log _{10}(e) \times 0.41\right)^{2}+\left(2 \times \log _{10}(e) \frac{\Delta d}{d}\right)^{2}
$$

where $\Delta l_{\text {Lee }}^{+}$and $\Delta l_{\text {Lee }}^{-}$are the positive and negative uncertainties on $l_{\text {Lee }}$ and where the second term accounts for the $41 \%$ relative uncertainty $\Delta d_{\text {Lee }} / d_{\text {Lee }}$ on the distance used in [7] and that we replaced with our own uncertainties on the distance $\Delta d / d$.

\subsection{X-ray-radio relation}

Using data from the $38 \mathrm{MSPs}$ detected in X-ray and in radio at 1400 or $400 \mathrm{MHz}$, we computed the best fit coefficient of a linear relation between the $\log _{10}$ of the X-ray and the radio luminosity of MSPs [10, 15]. The correlation coefficient between those two sets of variables is 0.456 . We perform a fit using Orthogonal Distance Regression from $\mathrm{SciPy}^{10}$, with weights $1 / \Delta l_{R}^{2}$ and $1 / \Delta l_{X}^{2}$. We found the following relation:

$$
l_{R}=(0.88 \pm 0.21) l_{X}+(-25.13 \pm 6.29) .
$$

In Figure 1, we show this relation together with the data used for the computation. The X-ray luminosity in erg/s is given by $L_{X}=10^{l_{X}}$ while the radio luminosity density in $\mathrm{mJy} \mathrm{kpc}^{2}$ is $L_{R}=10^{l_{R}}$.

\section{Prospects and conclusion}

In our previous study, we showed that the Chandra X-ray data were not inconsistent with a bulge population of MSPs, causing the Fermi GeV excess. We also selected promising MSPs candidates among the Chandra unidentified sources. The present work focused on this selection. First, thanks to multi-wavelength cross-matches, we excluded sources with possible UV or IR counterparts. We emphasize that for excluded sources, our goal was not to find their true counterpart, but rather

\footnotetext{
${ }^{10}$ https://docs.scipy.org/doc/scipy/reference/odr.html
} 


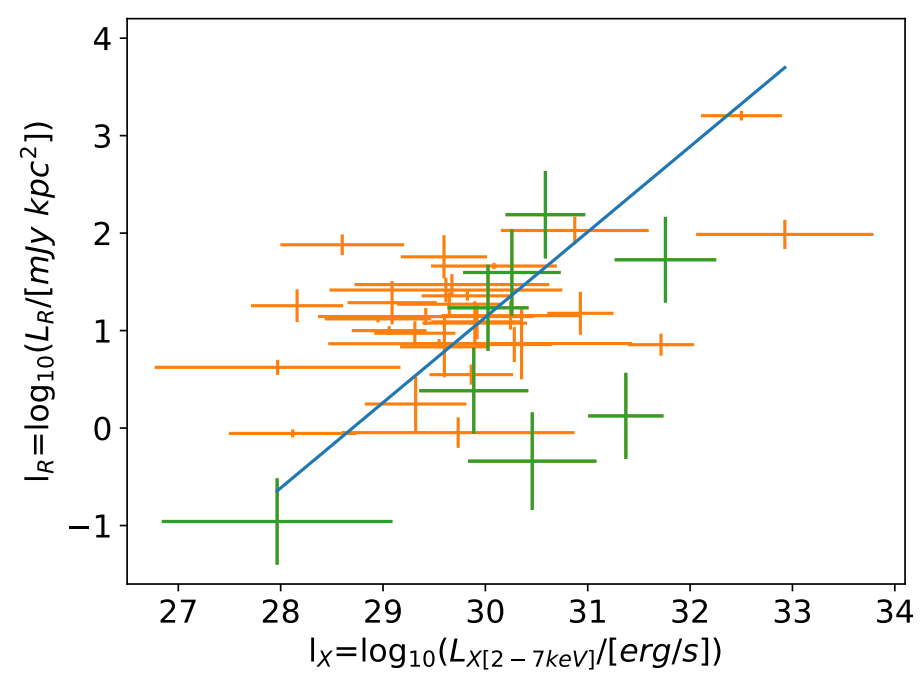

Figure 1: Relation between the X-ray and radio luminosity of MSPs given by equation 8 (blue line) constructed from data including MSPs with radio measurement at $1400 \mathrm{MHz}$ (orange) and at $400 \mathrm{MHz}$ only (green).

to find any possible counterpart, only relying on the angular separation. We do not aim at being conservative here. In other words, we want to be sure that the sources we keep as promising candidates have no counterpart at all in the existing data. Consequently, our selection of Chandra sources represents an interesting astrophysical population whether or not it is made of MSPs, as it only shines in $\mathrm{X}$ rays.

Second, we found a relationship between the X-ray luminosity and the radio luminosity of MSPs, using observational data. The next step would be to use this relation, given by equation 8 , to predict the radio luminosity of our MSPs candidates. However, for these sources, the unabsorbed $\mathrm{X}$-ray luminosity $L_{X}$, is not known. What is listed in the Chandra catalog is their absorbed flux, in different energy bands. The conversion from these fluxes to the unabsorbed X-ray luminosity highly depends on the distance and the X-ray spectral index of the sources. Nevertheless, we plan to retrieve this luminosity from the limited X-ray information that we have for each source, and from statistical properties we got from our MSP population simulation.

\section{References}

[1] Berteaud, J., Calore, F., Clavel, M. et al. 2020, arXiv:2012.03580

[2] Bailer-Jones, C. A. L., Rybizki, J., Fouesneau, M., et al. 2021, The Astronomical Journal, 161, 147. doi:10.3847/1538-3881/abd806

[3] Bartels, R. T., Edwards, T. D. P., Weniger, C. 2018, Monthly Notices of the Royal Astronomical Society, doi:10.1093/mnras/sty2529

[4] Benjamin, R. A., Churchwell, E., Babler, B. L., et al. 2003, The Publications of the Astronomical Society of the Pacific, 115, 953. doi:10.1086/376696 
[5] Greiss, S., Steeghs, D., Jonker, P. G., et al. 2014, Monthly Notices of the Royal Astronomical Society, 438, 2839. doi:10.1093/mnras/stt2390

[6] Jonker, P. G., Torres, M. A. P., Hynes, R. I., et al. 2014, The Astrophysical Journal Supplement, 210, 18. doi:10.1088/0067-0049/210/2/18

[7] Lee, J., Hui, C. Y., Takata, J. et al. 2018, The Astrophysical Journal, doi:10.3847/15384357/aad284

[8] Lin, D., Webb, N. A. and Barret, D. 2012, The Astrophysical Journal. doi:10.1088/0004$637 \mathrm{X} / 756 / 1 / 27$

[9] Maccarone, T. J., Torres, M. A. P., Britt, C. T., et al. 2012, Monthly Notices of the Royal Astronomical Society, 426, 3057. doi:10.1111/j.1365-2966.2012.21782.x

[10] Malov, I. F. \& Timirkeeva, M. A. 2019, Monthly Notices of the Royal Astronomical Society, 485, 5319. doi:10.1093/mnras/stz612

[11] Manchester, R. N., Hobbs, G. B., Teoh, A., et al. 2005, The Astronomical Journal, 129, 1993. doi: $10.1086 / 428488$

[12] Minniti, D., Lucas, P. W., Emerson, J. P., et al. 2010, New Astronomy, 15, 433. doi:10.1016/j.newast.2009.12.002

[13] Page, M. J., Brindle, C., Talavera, A., et al. 2012, Monthly Notices of the Royal Astronomical Society, 426, 903. doi:10.1111/j.1365-2966.2012.21706.x

[14] Skrutskie, M. F., Cutri, R. M., Stiening, R., et al. 2006, The Astronomical Journal, 131, 1163. doi: $10.1086 / 498708$

[15] Tudor, V., Miller-Jones, J. C. A., Patruno, A., et al. 2017, Monthly Notices of the Royal Astronomical Society, 470, 324. doi:10.1093/mnras/stx1168

[16] van Leeuwen, F., de Bruijne, J., Babusiaux, C., et al. 2021, Gaia EDR3 documentation, European Space Agency; Gaia Data Processing and Analysis Consortium.

[17] Yao, J. M., Manchester, R. N., \& Wang, N. 2017, The Astrophysical Journal, 835, 29. doi:10.3847/1538-4357/835/1/29 